P8 (continued)

(mean $=2.02 \pm 1.26 \mathrm{SD}$; 5-point scale). Content analysis of the coaching calls revealed many parents offered preschoolers SSBs as a treat or reward. After reading the Best Drinks guide, many planned to limit children's SSB consumption and offer more milk or water. Some planned to begin diluting children's juice, store SSBs in ways that deter kids from serving themselves, and use decorative cups or straws to make drinking healthier beverages fun. Parents felt limiting SSB in their family was very important (mean $=9.45 \pm 0.91 S D, 10$-point scale). Goals set by many parents after reading the guide were to limit SSB intake, increase milk and water intake, and communicate the importance of decreasing SSB with spouses and caregivers. At later coaching calls, most reported successfully meeting SSB goals.

Conclusion and Implications: Best Drinks for Families guide coupled with a brief coaching call has the potential to successfully help parents decrease SSB intake.

Funding: NIFA, Academy of Nutrition and Dietetics Foundation Ann Hertzler Research Grant

\section{P9 Factors Associated With Mindful Food Parenting Practices}

Keiko Goto, PhD, kgoto@csuchico.edu, California State University-Chico 400, West 1st Street, Chico, CA 95929; S. Yang; R. Mendez; J. Giampaoli, PhD, RD; A. Wylie; B. Seipel, PhD

Objective: To examine factors associated with mindful food parenting practices.

Design, Setting, and Participants: A cross-sectional survey was conducted using a convenience sample of 236 parents of elementary school students primarily consisting of Hispanic and non-Hispanic white children.

Outcome Measures and Analysis: Mindful food parenting practices were assessed using a validated mindful food parenting questionnaire. Other parent food behavior questions included frequencies of involving children in planning, shopping and preparing meals; availability of high-calorie food, fruits and vegetables at home; and frequencies of parent cooking, meal commensality at home, and eating out. Demographic data, such as gender, ethnicity, education, age, place of birth, and language spoken at home, were also obtained. Statistical analysis included Independent t-tests, one-way ANOVA and Spearman's correlation.

Results: Mindful food parenting practice scores were significantly higher among participants who were female, born in Mexico, and had some high school or less education. Mindful food parenting practices were significantly associated with fruit availability at home and frequencies of involving children in planning and preparing meals. Frequencies of parent cooking and meal commensality were significantly correlated with mindful parenting practices. The availability of sweets and sugary beverages at home, as well as frequency of eating out, were inversely correlated with mindful food parenting practices.
Conclusions and Implications: Mindful food parenting practices may influence a child's food environment and eating behaviors at home.

Funding: USDA NIFA

\section{P10 Assessment of Dietary Intake of Children With Autism Spectrum Disorders (ASD) Enrolled in a Summer Camp}

Amber Haroldson, PhD, RD, arharoldson@bsu.edu, Ball

State University, 232 Applied Technology Building, 2000

West University Avenue, Muncie, IN 47306; H. Killion, BS;

A. Haroldson, PhD, RD; J. Kandiah, PhD, RD, CD;

C. McIntosh, EdD, MBA, RN; S. Titus Dieringer, PhD;

A. Mahon, PhD; D. McIntosh, PhD

Objective: The objective of this study was to determine foods commonly consumed by school-aged children with Autism Spectrum Disorder (ASD) attending a five-week summer camp.

Design, Setting, Participants, and Intervention: To evaluate usual intake, a content validated food frequency questionnaire was completed by parents of children with ASD aged 6-12 years $(n=21)$.

Outcome Measures and Analysis: Frequency of consumption was assessed for 11 different food categories. Foods commonly consumed and/or avoided, adherence to special diets, and vitamin and mineral supplement usage were also recorded.

Results: Results suggest campers commonly consume 1-2 servings/day of fruits, vegetables, meat, other protein sources, sweets, fats, sugar-sweetened beverages, and snacks. Dairy and grains were the favored food groups, with common intakes of 3-4 servings/day. The majority of the subjects $(85.7 \%)$ did not adhere to any special diet (i.e., gluten-free, and/or casein-free). In addition to dietary intake, $43 \%$ of participants recorded regular intake of a vitamin or mineral supplement. Fruit and vegetable intakes were below the recommended daily amounts for this age group, with $76.2 \%$ and $90.5 \%$ of participants consuming $\leq 2$ servings of fruits and vegetables per day, respectively. Additionally, the daily intakes of sweets, fats, sugar sweetened beverages and snacks were above recommendations for this age group.

Conclusions and Implications: Future research and nutrition intervention programs involving children with ASD should focus on intervention efforts to increase fruit and vegetable intake and decrease high-fat, high-sugar, and snack food consumption.

Funding: None

\section{P11 "We Don't Snack": The Routine Behavior of Feeding Children and 10 0'Clock and 4 0'Clock}

Emma Jacquier, BS, MSc, emma.jacquier@yahoo.com, Lancaster University, 12 Vreeland Road, Bailrigg, Lancaster LA1 4YW, Canada; A. Gatrell 